\title{
SELECTED SPIN PHYSICS RESULTS FROM COMPASS, HERMES AND RHIC
}

\author{
KLAUS RITH \\ Physikalisches Institut, Universität Erlangen-Nürnberg \\ Erwin-Rommel-Str. 1, 91058 Erlangen, Germany \\ E-mail: klaus.rith@physik.uni-erlangen.de
}

\begin{abstract}
This review summarizes recent experimental results in polarized lepton-nucleon and proton-proton scattering.

PACS: $12.38 . Q k, 13.60 .-r, 13.60 . H b, 13.87 .-\mathrm{a}, 13.88+\mathrm{e}, 14.20 . \mathrm{Dh}$. 14.65.-q, 24.70.+s
\end{abstract}

\section{INTRODUCTION}

The spin structure of the nucleon and the decomposition of the nucleon spin into contributions from quark and gluon spins and quark and gluon orbital angular momenta has already been thoroughly discussed in the review by X. Ji [1]. Therefore I will not address these general aspects but concentrate on the discussion of some recent spin physics results from five experiments: COMPASS at CERN, HERMES at HERA/DESY and BRAHMS, PHENIX and STAR at RHIC/BNL. I will omit the interesting topic of exclusive processes and Generalized Parton Distributions as this has been nicely covered in the review by M. Garcon [2]. Before I discuss the new results let me briefly summarize the main features of these experiments.

The COMPASS experiment [3] uses the CERN high-energy muon beam $\left(E_{\mu} \approx 160\right.$ $\mathrm{GeV}$, beam polarization $\left.P_{B} \approx-0.76\right)$, and a cryogenic polarized LiD target $\left(P_{T}^{D} \approx 0.5\right)$ with both longitudinal and transverse target polarization. Advanced technology is used for many detectors and the data acquisition system. COMPASS started data taking in summer 2002.

HERMES [4] uses the longitudinally polarized electron or positron beam of HERA ( $E \approx 27.5 \mathrm{GeV}$, beam polarization $P_{B, \max } \approx 0.6$ ) and for the spin program polarized pure atomic gas targets of hydrogen, deuterium $\left(P_{T}^{H, D} \approx 0.85\right)$ and ${ }^{3} \mathrm{He}\left(P_{T} \approx 0.5\right)$, internal to the electron storage ring. In the years 1995-2000 data were taken with longitudinal target polarization. A review of the results obtained with longitudinally polarized targets can be found in [5]. In 2002-05 a transversely polarized hydrogen target has been used.

Polarized proton-proton collisions provide an additional powerful experimental tool to study the spin structure of the nucleon. RHIC, equipped with a system of Siberian Snakes, is the first facility of colliding high energy polarized protons, so far at a center of mass energy of $\sqrt{s}=200 \mathrm{GeV}$. Spin rotator magnets upstream and downstream of the STAR and PHENIX interaction regions provide longitudinal beam polarization for these experiments, while at BRAHMS only transverse beam polarization is possible. Many details about RHIC and the experiments can be found in Ref. [6]. Enormeous progress 


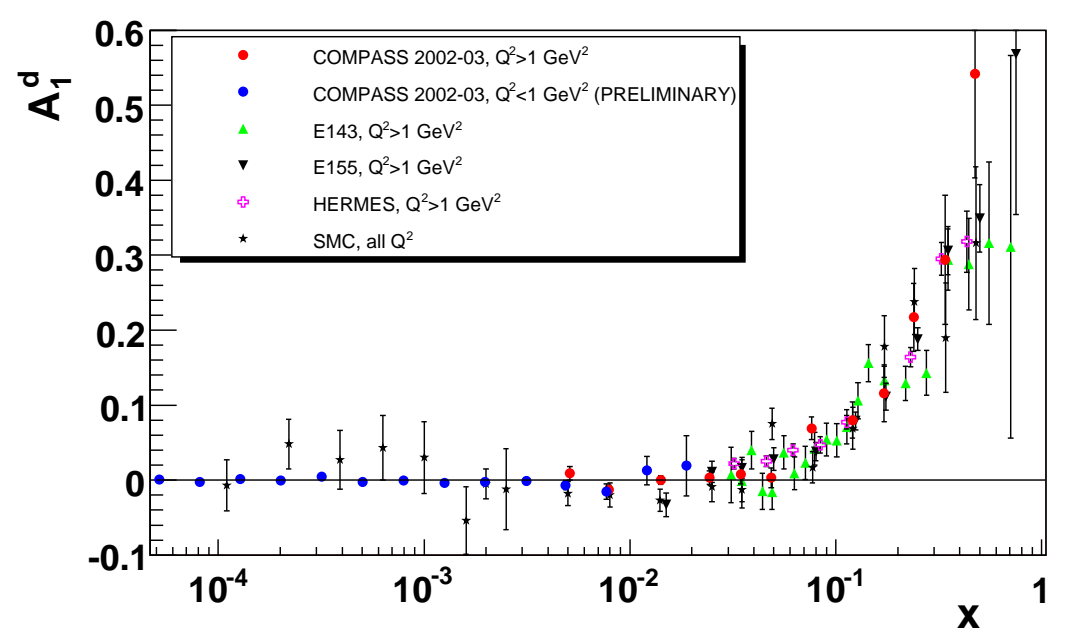

FIGURE 1. COMPASS compilation of the world data for the virtual photon asymmetry $A_{1}^{d}(x)$.

has been made for the spin programme at RHIC. Recently peak beam polarizations of about 0.67 have been obtained, reaching essentially the design goal of 0.7 , and peak luminosities of $2.5 \times 10^{31} \mathrm{~cm}^{-2} \mathrm{~s}^{-1}$, which are still somewhat lower than the design value. I want to use this opportunity to congratulate the RHIC colleagues for these impressive achievements.

\section{DOUBLE-SPIN VIRTUAL-PHOTON ASYMMETRIES AND NLO-QCD ANALYSIS}

New preliminary results for the virtual-photon double-spin asymmetry $A_{1}(x)$ and the spin structure function $g_{1}(x)$ are available from the COMPASS 2002-2003 deuteron data, which supersede previous results [7]. HERMES has previously published results for $g_{1}^{n}$ [8] and $g_{1}^{p}$ [9]. The final analysis of all data taken with longitudinally polarized hydrogen and deuterium targets will be published soon [10]. Fig. 1 shows the recent COMPASS compilation of world data for $A_{1}^{d}(x)$ which also contains preliminary results for the 2002-03 data sample at $Q^{2}<1(\mathrm{GeV} / \mathrm{c})^{2}$. Note the huge increase in precision of the COMPASS results compared to SMC at low $\mathrm{x}$.

HERMES data at low $\mathrm{x}$ indicate that $A_{1}^{p}$ is compatible with zero below $x=0.01$ while $A_{1}^{d}$ vanishes already below $x=0.05$. Under the assumption that the first moment of $g_{1}^{d}$ saturates for $x<0.04$ it can be deduced [10] that $\Delta \Sigma$, the fraction of the nucleon spin which can be attributed to quark spins, is about $0.35 \pm 0.03$. The new COMPASS data at $Q^{2}<1(\mathrm{GeV} / \mathrm{c})^{2}$ cover an x-range from about $2 \times 10^{-2}$ down to about $5 \times 10^{-5}$, where $<Q^{2}>\simeq 7 \times 10^{-3}(\mathrm{GeV} / \mathrm{c})^{2}$. The asymmetry is compatible with zero over essentially the whole $x$ range. Possibly the vanishing asymmetry is due to a large unpolarized soft background. The low $Q^{2}$ values of these very precise and interesting inclusive data hardly allow to interpret them in the framework of perturbative QCD and to extract informations about the quark helicity distributions at low $\mathrm{x}$. 
Several groups have performed NLO QCD-fits to the spin-dependent asymmetries or structure functions, respectively (see e.g. the more recent papers $[11,12,13,14,15]$ ). The up-quark helicity distribution $\Delta u(x)$ seems to be rather well determined from the QCD fits as well in shape as in magnitude, while the fits give a much larger possible range for $\Delta d(x)$. The sea-quark helicity distributions and especially $\Delta s(x)$ are essentially undetermined from these fits. All authors obtain a negative small sea-polarization, a relatively small contribution of quark spins to the spin of the nucleon, $\Delta \Sigma \approx 0.25$, and a positive moment of the gluon helicity distribution $\Delta g \approx 0.5$. The results for $\Delta \Sigma$ from the NLO QCD fits tend to be systematically smaller by about $0.1-0.15$ than those extracted from the data of individual experiments [10] in the measured x-range. Correspondingly $g_{1}^{d}$ should become negative and should reach very large values of about -(30-40) in the unmeasured low $\mathrm{x}$ region. This is, of course, not impossible, but to my taste rather unlikely. I personnally believe, however, that part of this discrepancy might be due to the functional form used for the parton distributions in these analyses which, for the given constraints, forces them to become negative at low $\mathrm{x}$. The errors of the fits for $\Delta g(x)$ are large, and this important quantity is essentially unconstrained by the present inclusive data on polarized DIS. Low-x data are urgently needed and I hope that our dream of a polarized electron-proton collider like e-RHIC will become reality in the next decade.

\section{QUARK HELICITY DISTRIBUTIONS}

The quark helicity distributions can in principle be determined in polarized semiinclusive deep-inelastic scattering when in addition to the scattered lepton also a leading hadron is detected which provides a statistical tag on the struck quark flavor. Results of such analyses have been reported by SMC [16] for leading hadrons and by HERMES $[17,18,19]$ for identified positive and negative pions and kaons.

The helicity distributions $\Delta u(x)$ and $\Delta d(x)$ extracted from the HERMES data are consistent with previous results and with the LO-QCD fits. The sea-quark helicity distributions $\Delta^{-} u(x), \bar{\Delta}(x)$ and $\Delta s(x)$, extracted separately from these data for the first time, are consistent with zero and with each other. The statistical uncertainties are, however, still substantially larger than the magnitude of the distributions obtained from the QCD fits. Within errors there is no indication for a substantial negative polarization of the strange sea that was deduced from the analysis of inclusive data assuming SU(3) symmetry. There is also no evidence in the data for a large flavor asymmetry in the light quark sea helicity distribution $\Delta^{-} u(x)-\bar{d}(x)$. More high precision data are urgently needed to better constrain the sea-quark helicity distributions. The future RHIC measurements of single-spin asymmetries in $\vec{p}+p \rightarrow W^{ \pm} \rightarrow l^{ \pm} v$ at $\sqrt{s}=500 \mathrm{GeV}$, which are scheduled to start in 2009, will be of special importance, since for an integrated luminosity of $800 \mathrm{pb}^{-1}$ the anticipated statistical accuracy of the up- and down-quark and antiquark distributions will be about a factor of 10 better than the accuracy of the HERMES data. 


\title{
THE GLUON HELICITY DISTRIBUTION $\Delta g\left(x_{g}, Q^{2}\right)$
}

\author{
$\Delta g\left(x_{g}, Q^{2}\right)$ from polarized lepton-nucleon scattering
}

The most promising approach for studying $\Delta g$ is the direct measurement via processes where the gluon density enters the cross section directly. In lepton-nucleon scattering such a process is photon-gluon fusion (PGF) $\left(\gamma^{*} g \rightarrow q^{-} q\right)$. This process can be isolated either by studying reactions in which a charmed quark anti-quark pair is produced and the necessary hard scale is given by the mass of the c-quark, or alternatively by measuring a quark and an anti-quark jet or pairs of hadrons, respectively, at large transverse momenta $p_{T}$ relative to the direction of the virtual photon. To relate the measured double-spin asymmetry (DSA) for pairs of high- $p_{T}$ hadrons to the gluon helicity distribution $\Delta g\left(x_{g}, Q^{2}\right)$ or the gluon polarization $\left(\Delta g / g\left(x, Q^{2}\right)\right)$, respectively, one has to understand precisely the relative contributions of the various subprocesses to the hadron pair production cross section and their variation with $p_{T}$. This requires a detailed Monte Carlo simulation. The situation is less complicated for the determination of $\Delta g\left(x_{g}, Q^{2}\right)$ from the DSA of open charm production which is dominated by PGF. This process suffers, however, from the relatively low cross section.

Results for the DSA for the production of pairs of high- $p_{T}$ hadrons have been published by HERMES [20] for quasi-real photoproduction, by SMC [21] for DIS events with $Q^{2}>1(\mathrm{GeV} / \mathrm{c})^{2}$, and for a subsample of quasi-real photoproduction data collected in 2002-2003 by COMPASS [22, 23]. The COMPASS 2004 data doubled the available statistics. Preliminary COMPASS results [24] for high- $P_{T}$ hadron pairs with $Q^{2}>1$ $(\mathrm{GeV} / \mathrm{c})^{2}$ from the 2002-03 data and for quasi-real photoproduction of hadron pairs and charm production from the total statistics collected in 2002-04 are shown in Fig. 2 together with the previous determinations and are compared to three parameterizations from NLO QCD fits based on Ref. [13] corresponding to values for $\Delta g$ of 0.16 (GRSVmin), 0.62 (GRSV-std) and 2.5 (GRSV-max).

It is difficult to draw a firm conclusion from these results. A large positive gluon distribution is definitively excluded, the high precision quasi-real photoproduction results from COMPASS either favour a very small $\Delta g$, or $\Delta g\left(x_{g}\right)$ has a node at $x_{g}$ around 0.1 .

\section{$\Delta g\left(x_{g}, Q^{2}\right)$ from polarised proton-proton scattering}

Double-spin asymmetries in polarised proton-proton scattering are the second tool to gain direct information about the gluon helicity distribution. Several different reaction channels can be studied. Examples are: inclusive jet production $(\vec{p} \vec{p} \rightarrow$ jet $+X)$, where the dominant partonic subprocesses are $(\vec{g} \vec{g} \rightarrow g g)$ and $(\vec{g} \vec{q} \rightarrow g q)$, inclusive pion production $(\vec{p} \vec{p} \rightarrow \pi+X)$ with the same subprocesses, direct photon production $(\vec{p} \vec{p} \rightarrow \gamma+X, \gamma+j e t+X)$ corresponding to $(\vec{g} \vec{q} \rightarrow \gamma q)$ and heavy quark production $(\vec{p} \vec{p} \rightarrow D+X, B+X)$, corresponding to $\left(\vec{g} \vec{g} \rightarrow c{ }^{-} c, b \bar{b}\right)$. These reactions involve a convolution of two parton distributions and the interpretation of the results is therefore always based on a comparison to a Monte Carlo simulation. 


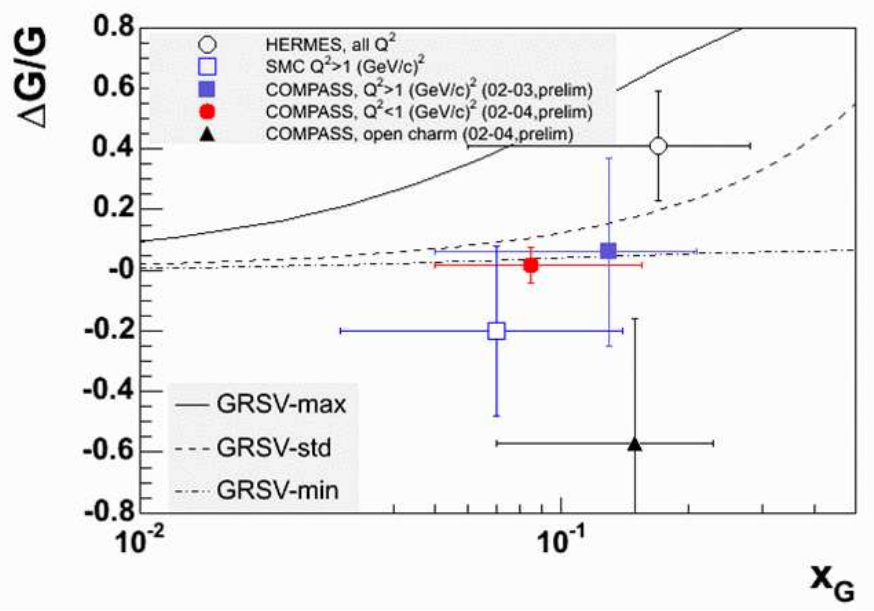

FIGURE 2. COMPASS compilation of results for the gluon polarizations from deep-inelastic polarized lepton-nucleon scattering as a function of $x$.

STAR results on $A_{L L}(j e t)$ : The STAR experiment reported the first measurement of the longitudinal DSA for inclusive jet production $[25,26]$ at $\sqrt{s}=200 \mathrm{GeV}$ and mid-rapidity. Fig. 3 (left panel) shows the result from the 2003-04 data set. The curves show theoretical calculations $[27,28]$ at equal factorization and renormalization scales $\mu \equiv \mu_{F}=\mu_{R}=p_{T}$ for the parton helicity distributions of Ref. [13] for different assumptions of the shape and magnitude of the gluon helicity distribution at the initial scale $Q_{0}^{2}=0.4(\mathrm{GeV} / \mathrm{c})^{2}$ in the analysis. The measured asymmetries are systematically below the expectation for $\Delta g\left(x_{g}, Q_{0}^{2}\right)=g\left(x_{g}, Q_{0}^{2}\right)$ and thus disfavor a large positive gluon polarisation. They are consistent with the other evaluations $\Delta g\left(x_{g}, Q_{0}^{2}\right)=0$ and $\Delta g\left(x_{g}, Q_{0}^{2}\right)=-g\left(x_{g}, Q_{0}^{2}\right)$.

PHENIX results on $A_{L L}\left(\pi^{0}\right)$ : The second piece of information comes from the PHENIX collaboration which measured the longitudinal DSA $A_{L L}\left(\pi^{0}\right)$ in inclusive $\pi^{0}$ production at mid-rapidity and $\sqrt{s}=200 \mathrm{GeV}$. At the kinematics of this experiment the relative subfractions of the contributing processes vary substantially with $p_{T}$ [27]. The preliminary results from Run 5, which supersede the published result [29], are shown in the right panel of Fig. 3 together with different expectations based on the GSRV parton distributions. The measured asymmetry is small with an average value around zero. Again GSRV-max is excluded by these data. To quantify the agreement/disagreement with the different expectations and the data the collaboration gives confidence levels. These are about $20 \%$ for GSRV-std and GSRV- $(\Delta g=0)$, and essentially zero for the other two expectations.

My main conclusions are: the new results indicate that $\Delta g / g$ might be rather small. Much higher precision data from different processes are needed to settle the question about shape and magnitude of $\Delta g\left(x_{g}\right)$. RHIC data at higher values of $p_{T}$, which can be expected from future high luminosity runs at $\sqrt{s}=500 \mathrm{GeV}$ will play a crucial role. 


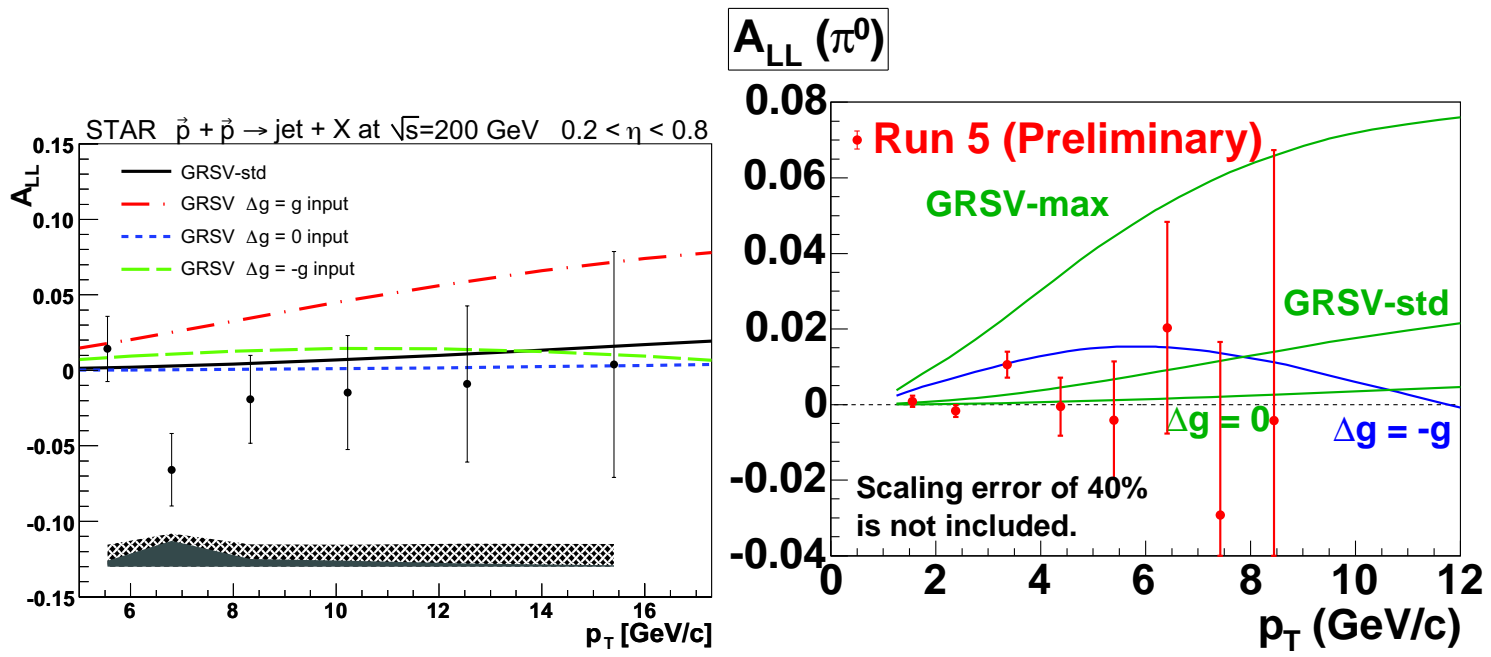

FIGURE 3. STAR results for the longitudinal double-spin asymmetry for inclusive jet production as a function of $p_{T}$ (left panel) and preliminary PHENIX results for the longitudinal double-spin asymmetry for inclusive $\pi^{0}$ production as a function of $p_{T}$ (right panel) at mid-rapidity and $\sqrt{s}=200 \mathrm{GeV}$.

\section{TRANSVERSE SPIN PHYSICS}

\section{Transversity, Collins and Sivers Effect}

A complete description of the partonic structure of the nucleon in leading twist requires in addition to the unpolarized quark distributions $q\left(x, Q^{2}\right)$ and the quark helicity distributions $\Delta q\left(x, Q^{2}\right)$ a third quark distribution, named transversity $\delta q\left(x, Q^{2}\right)$ [30], which survives integration over intrinsic transverse momenta.

Very little experimental information about transversity exists up to know. It is a chiralodd quantity and cannot be probed in inclusive DIS since hard interactions conserve chirality. Its measurement requires processes involving some additional chiral-odd structure like, e.g., another transversity distribution in transversely polarized Drell-Yan production of lepton pairs, or a chiral-odd fragmentation function in semi-inclusive DIS. One of the possibilities to study transversity is via the azimuthal angular asymmetry in the distribution of hadrons produced in semi-inclusive DIS. Such an asymmetry can arise if the transverse polarization of the struck quark influences the transverse momentum of the produced hadron and thereby its distribution in the azimuthal angle $\phi$ about the virtual photon direction relative to the lepton scattering plane. The corresponding chiral-odd fragmentation function $H_{1}^{\perp}$, which is also odd under naive time reversal, is known as the "Collins fragmentation function" [31, 32].

Such an angular asymmetry could, however, also be produced by a different mechanism involving correlations between the transverse polarization of the target nucleon and the transverse momentum of quarks. The transverse quark momentum can survive both the photo-absorption and the fragmentation and can reappear in the transverse momentum of the produced hadron and thereby influence its azimuthal distribution relative to the direction of target polarization. The corresponding "Sivers distribution function" 

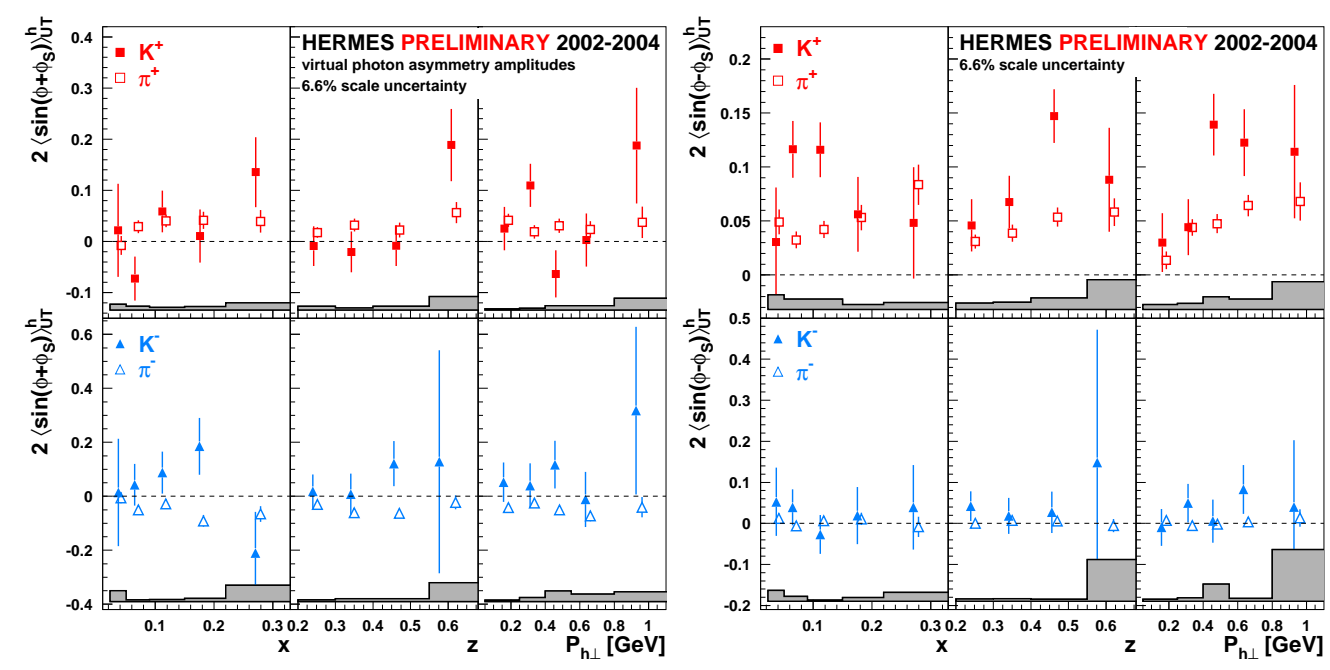

FIGURE 4. HERMES results for the "Collins moments" (left panel) and the "Sivers moments" (right panel) for charged pions and kaons obtained with a transversely polarised hydogen target.

$f_{1 T}^{\perp}[33,34]$ is time-reversal odd. Its measurement therefore requires initial or final state interactions. One especially interesting aspect of this distribution function is the possible relation to orbital angular momenta of quarks [35].

Measurements with a transversely polarized target allow to distinguish between the Collins mechanism and the Sivers mechanism $[36,37,38]$. The Collins mechanism will cause a $\sin \left(\phi+\phi_{S}\right)$ moment proportional to $\delta q(x) \cdot H_{1}^{\perp}(z)$, while the Sivers mechanism will cause a $\sin \left(\phi-\phi_{S}\right)$ moment proportional to $f_{1 T}^{\perp}(x) \cdot D(z)$. Here $\phi_{S}$ is the angle between the electron scattering plane and the target spin axis and $D(z)$ is the familiar unpolarized fragmentation function.

In the years 2002-05 HERMES has taken data with a transversely polarised proton target and COMPASS has taken some fraction of the data with a transversely polarised deuteron target. HERMES has evaluated the corresponding azimuthal asymmetry, in two dimensions and extracted the Collins and Sivers virtual-photon asymmetries by a simultaneous fit to $\sin \left(\phi+\phi_{S}\right)$ and $\sin \left(\phi-\phi_{S}\right)$.

Preliminary results for the "Collins moments" for charged pions and kaons, based on the data taken in the years 2002-04 [39, 40], which correspond to about 30\% of the total statistics and supersede the published result for charged pions [41], are shown in the left panel of Fig. 4. The measured asymmetries are small but different from zero providing evidence for the existence of both the transversity distribution and the Collins fragmentation function.

In the right panel of Fig. 4 the preliminary HERMES results from the 2002-04 data sample for the "Sivers moments" are shown. The $\pi^{+}$and $K^{+}$asymmetries are significantly positive, providing the first evidence for a T-odd parton distribution function appearing in leptoproduction. Consequently one has to conclude from this result that orbital angular momenta of quarks inside the nucleon are non-zero. At present it is, however, not jet possible to quantitatively relate the magnitude of this asymmetry to the fraction of nucleon spin which can be attributed to orbital angular momenta of quarks. 

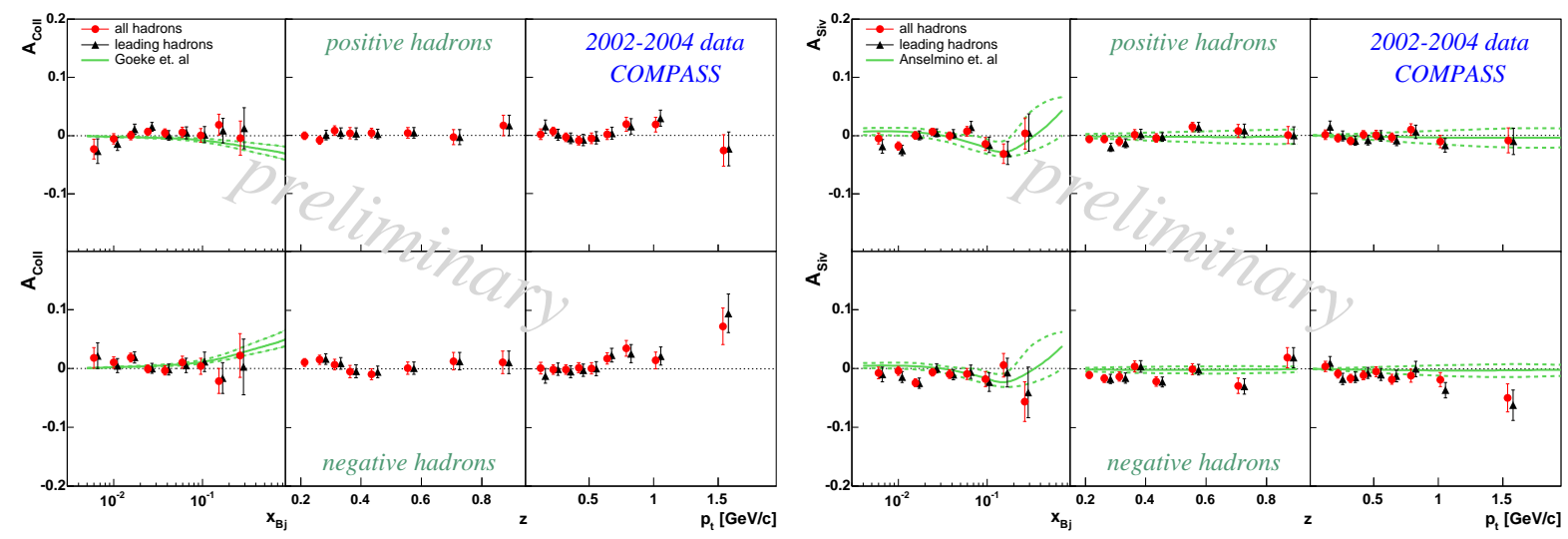

FIGURE 5. COMPASS results for the Collins moments (left panel) and Sivers moments (right panel) of positive and negative hadrons obtained with a transversely polarized deuterium target.

The positive kaon amplitudes are about twice as large as the pion amplitudes, which might point to a large Sivers function for sea-quarks.

COMPASS extracted the Collins and Sivers asymmetries for both positive and negative hadrons from the combined 2002-04 deuteron data in an 1-dimensional analysis. Preliminary results are shown in Fig. 5 together with theoretical predictions [42, 43]. Statistical errors have been reduced by at least a factor of two compared to the previously published results [44]. Note that COMPASS and HERMES use a different convention for the definition of the angle $\phi$ which differs by $\pi$ and therefore the sign of one of the data sets should be inverted for a direct comparison. The COMPASS data are compatible with zero within errors. Very likely this can be traced back to cancellations arising due to the use of a deuteron target.

\section{Transverse Single-Spin Asymmetries in Proton-Proton scattering}

The transverse single-spin asymmetry or analyzing power $A_{N}$ in proton-proton scattering is $A_{N}=\left(\sigma^{+}-\sigma^{-}\right) /\left(\sigma^{+}+\sigma^{-}\right)$, where $\sigma^{+(-)}$is the cross section for $p_{\uparrow(\downarrow)}+p$ collisions with the beam or target spin oriented up or down transversely to the scattering plane. Large asymmetries of up to $30 \%$ for $\pi^{0}$ production have been measured already 15 years ago by E704 at FNAL [45] at $\sqrt{s} \sim 20 \mathrm{GeV}$ for relative large Feynman-X and moderate $p_{T}$. At that time it was expected from QCD that the cross sections should have very little spin dependence and that transverse asymmetries should be suppressed by $\alpha_{s} m_{q} / M$. The Sivers effect has been invented [34] to explain the observed large SSA. Recent theoretical attempts include the role of partonic transverse momentum effects, transversity distribution and Collins fragmentation, the Sivers effect, and higher-twist effects arising from quark-gluon correlations [46, 47, 48, 49]. Sizeable transverse SSAs for $\pi^{0}$ production at $\sqrt{s}=200 \mathrm{GeV}$ have been recently reported by STAR $[50,51]$ and for charged pions and kaons, protons and antiprotons by BRAHMS [52, 53]. The preliminary BRAHMS results for the analyzing power $A_{N}$ for these charged hadrons is shown in Fig. 6. The $A_{N}$ values are positive for $\pi^{+}$and negative for $\pi^{-}$, they are positive for 


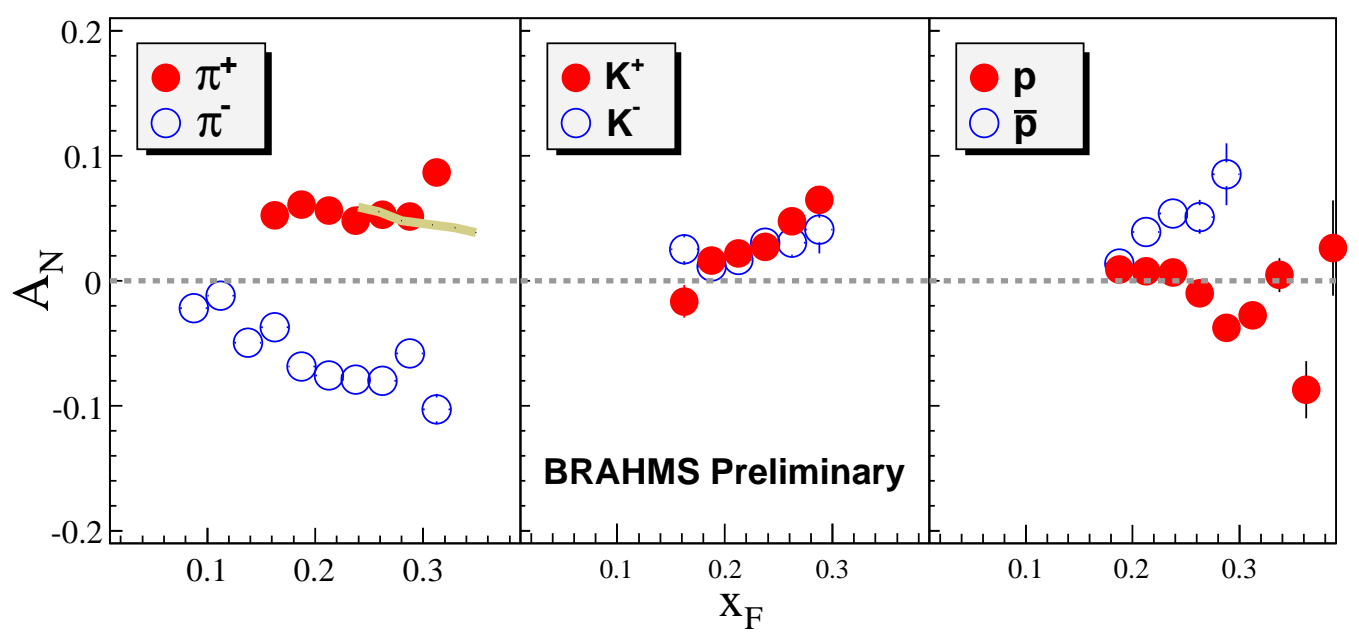

FIGURE 6. Preliminary BRAHMS results for the analyzing power $A_{N}$ for charged pions, kaons, protons and antiprotons.

both $\mathrm{K}^{+}$and $\mathrm{K}^{-}$and for antiprotons, while they are compatible with zero for protons. Especially interesting is the non-zero asymmetry measured for negative kaons and for antiprotons, since they indicate, similar to the large $K^{+}$Sivers asymmetry measured by HERMES, that sea-quarks may play an important role for the origin of such transverse asymmetries. The data call for more theoretical modeling with a good understanding of the fragmentation process.

\section{CONCLUSIONS AND OUTLOOK}

During the last year exciting new information on the nucleon spin structure from polarized lepton-nucleon and proton-proton scattering has been obtained. Longitudinal double-spin asymmetries for pairs of high- $p_{T}$ hadrons and charm events in semiinclusive DIS and for $\pi^{0}$ - and jet production in pp scattering indicate that the gluon helicity distribution might be quite small. Non/zero single-spin azimuthal asymmetries from transversely polarized targets and the analyzing power $A_{N}$ provide evidence for the existence of the transversity and Sivers distribution functions and the Collins fragmentation function. Many more interesting results can be expected in the near future.

\section{ACKNOWLEDGEMENTS}

I would like to thank the organisers for the invitation to deliver this plenary talk and G. Bunce, M. Grosse-Perdekamp, C.A. Gagliardi, R. Joosten, J.H. Lee and A. Magnon for providing me with plenty of material for the presentation. 


\section{REFERENCES}

1. X. Ji, these proceedings.

2. M. Garcon, these proceedings

3. G.K. Mallot for the COMPASS collaboration, Nucl. Instr. Meth. A 518, 121-124 (2004).

4. K. Ackerstaff et al. (HERMES), Nucl. Instr. Meth. A 417, 230-265 (1998).

5. K. Rith, Progr. Part. Nucl. Phys. 49, 245-324 (2002).

6. Special Issue: RHIC and Its Detectors, Nucl. Instr. Meth. A 499 (2003).

7. E.S. Ageev et al. (COMPASS), Phys. Lett. B 612, 154-164 (2005).

8. K. Ackerstaff et al. (HERMES), Phys. Lett. B 404, 383-389 (1997).

9. A. Airapetian et al. (HERMES), Phys. Lett. B 442, 484-492 (1998).

10. A. Airapetian et al. (HERMES), in preparation.

11. J. Blümlein and H. Böttcher, Nucl. Phys. B 636, 225-263 (2002); J. Phys. G 29, 1959-1961 (2003).

12. D. de Florian et al., AIP Conf. Proc. 792, 921-924 (2005).

13. M. Glück et al., Phys. Rev. D 63, 094005 (2001).

14. M. Hirai et al., Phys. Rev. D 74, 014015 (2006).

15. E. Leader et al., Phys. Rev. D 73, 034022 (2006).

16. B. Adeva et al. (SMC), Phys. Lett. B 420, 180-190 (1998).

17. K. Ackerstaff et al. (HERMES), Phys. Lett. B 464, 123-134 (1999).

18. A. Airapetian et al. (HERMES), Phys. Rev. Lett. 92, 012005 (2004).

19. A. Airapetian et al. (HERMES), Phys. Rev. D 71, 012003 (2005).

20. A. Airapetian et al. (HERMES), Phys. Rev. Lett. 84, 2584-2588 (2000).

21. B. Adeva et al. (SMC), Phys. Rev. D 70, 012002 (2004).

22. E.S. Ageev et al. (COMPASS), Phys. Rev. D 70, 012002 (2004).

23. E.S. Ageev et al. (COMPASS), Phys. Lett. B 633, 25-32 (2006).

24. E.M. Kabuss, these proceedings.

25. B.I. Abelev et al. (STAR), submitted to Phys. Rev. Lett, hep-ex/0608030.

26. K. Kowalik, these proceedings.

27. B. Jäger et al., Phys. Rev. D 70, 034010 (2004).

28. J. Pumplin et al., J. High Energy Phys. 0207, 012 (2002).

29. S.S. Adler et al.(PHENIX), Phys. Rev. Lett. 93, 202002 (2004); Phys. Rev. D 73, 091102 (2006).

30. V. Barone and P.G. Ratcliffe, Transverse Spin Physics, World Scientifi c, 2003.

31. J. Collins, Nucl. Phys. B 396, 161-182 (1993).

32. J. Collins et al., Nucl. Phys. B 420, 565-582 (1994).

33. P. J. Mulders and R. D. Tangerman, Nucl.Phys. B 461, 197-237 (1996); Erratum Nucl.Phys. B 484, 538-540 (1997).

34. D. W. Sivers, Phys. Rev. D 41, $83-90$ (1990); Phys. Rev. D 43, 261-263 (1991).

35. M. Burkardt, Phys. Rev. D 66, 114005 (2002), Phys. Rev. D 69, 074032 (2004).

36. M. Anselmino and F. Murgia, Phys. Lett. B 442, 470-478 (1998).

37. J. Collins, Phys. Lett. B 536, 43-48 (2002).

38. D. Boer et al., Nucl. Phys. B 667, 201-241 (2003).

39. U. Elschenbroich, PhD thesis, University of Gent (2006), DESY-THESIS-2006-004.

40. A. Miller, these proceedings.

41. A. Airapetian et al. (HERMES), Phys. Rev. Lett. 94, 012002 (2005).

42. A.V. Efremov et al., Phys. Rev. D 73, 094025 (2006).

43. M. Anselmino et al., Phys. Rev. D 72, 094007 (2005) Erratum Phys. Rev. D 72, 099903 (2005).

44. V.Yu. Alexakhin et al. (COMPASS), Phys. Rev. Lett. 94, 202002 (2005).

45. D.L. Adams et al.(FNAL/E704), Phys. Lett. B 264, 462 (1991).

46. J. Qiu and G. Sterman, Phys. Rev. D 59, 014004 (1999).

47. M. Anselmino et al., Phys. Lett. B 362164 (1995).

48. M. Anselmino et al., Phys. Rev. D 60, 054027 (1999).

49. Y. Koike, AIP Conf. Proc. 675, 449 (2003).

50. J. Adler et al. (STAR), Phys. Rev. Lett. 92, 171801 (2004).

51. C.A. Gagliardi, hep-ex/0607003.

52. F. Videbæk, hep-ex/0601008.

53. J.H. Lee, contribution to DIS06. 